\title{
Redwi Danjé Nan Zòn Pòt ${ }^{1}$
}

Hal S. Knowles, III, Kathleen C. Ruppert, Karla A. Lenfesty ak Barbara Haldeman²

Pòt bay bonjan aksè pou rentré ak sòti nan kay. Sepandan, gwo van kapab fé wout pou domaje siklòn.

\section{Ki Sa Ki Fè Youn Pòt Gen Sirté?}

Yon bon pòt genyen twa gon ak yon seri ki gen yon bout fè de youn pous. Bout fé-a gen pou li rantré nan ankadreman mi-an dèyè tèt pòt la.

Bon pòt egalman ouvri sou deyò kay la. Bon jan pòt gen pou fèt ak fè ouswa bwa ki solid, Mété panno sou pòt ki genyen nenpòt ki fenèt sou li.

\section{Ki Sak Ki Fè Pòt Doub Inik}

Pòt doub, kankou pòt Fransé bezwen plis pwoteksyon. Sa ki fè sa, se paske pòt la dé fwa pi laj ke pòt senp.

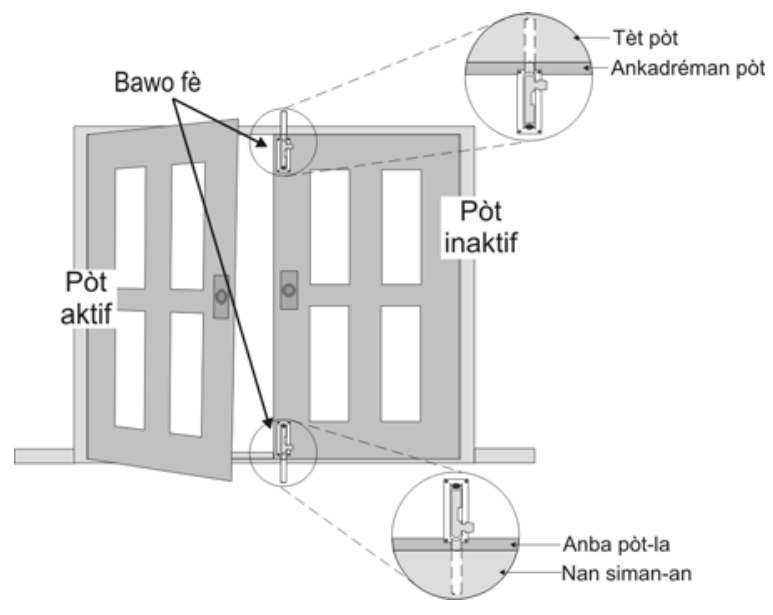

Nou gen dwa fè pòt doub-la plis rezistan nan van-an si nou mete yon bon jan bawo en fè sou bò ki pa ouvri-a. Bawo fè-a gen pou enstalé nan tèt ankadreman pòt-la rantré nan mi-a ak youn lòt ke ou mete anba pòt la atè-a nan siman-an.

\section{Pou Ki Sa Pòt Remize Pa Kenbé?}

Nòmalman pòt remize fèt ak panno fen tankou fèy tòl ki kouvri yon bon lajè pòt nan devan yon kay. Anba kondisyon lè gro van ap soufflé pòt sa-yo pa kapab kenbé.

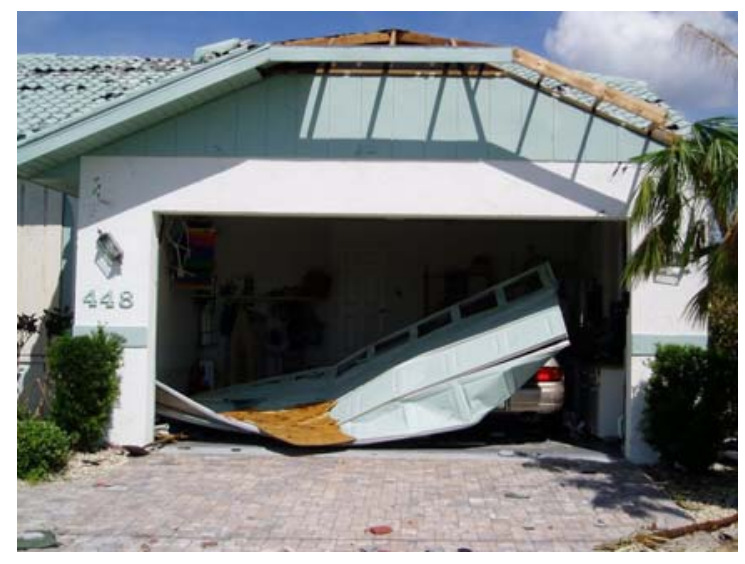

Sous: Pwogram Ki Monitè Kòt Laflorid

Gro van gen dwa kòz pòt remize-lan pou tonbé andedan kay-la ouswa sòti nan trak-li. Kou sa rive van gen dwa rantré andedan kay-la. Vanan lè sa-a kréye yon gro fòs andedan kay-la ki lè sa gen dwa raché fenèt, pòt, miray, ak dokay-la.

1. Dokiman sa-a se pati 5 dé 5 nan Edikasyon + Aksyon = Coleksyon Kontwol Dega Van. Dat piblikasyon: Novanm 2005.

2. Hal S. Knowles, III, Kòdinatè, Pwogram pou Resous Bon Jan Kominoté; Kathleen C. Ruppert, Syantis Estansyon Asosyé, Sévis Estansyon Enèji Laflorid; Karla A. Lenfesty, Espesyalis Kontwol Dega Van Siklòn, Estansyon Kopératif pou Konté St Lucie, Enstiti pou manjé ak Syans Agrikiltirèl, Inivvèsité Laflorid; Barbara Haldeman, Asistan Editè, Sèvis Estansyon Enéji Laflorid.

Enstiti pou manje ak Syans Agrikiltirèl (IMSA) se yon Opòtinité pou Anplwa Egal - Anplwayè Aksyon Afimatif gen dwa pou founi rechèch, enfòmasyon edikasyonèl ak lòt sèvis sèlman bay endividyèl ak enstitisyon kap fonksyoné san fè atansyon a ras, kwayans, koulè, relijyon, laj, enfimité, sex, oryantasyon sexsyèl, stati marital, orijin nasyonal, opinyon ou afilyasyon politik. Pou enfòmasyon sou kouman pou jwenn lòt piblikasyon estansyon, pran kontak ak Biro Sèvis Estansyon Koperatif ki bò Konté pa-ou. Sèvis Estansyon Koperatif Laflorid / Enstiti pou manjé ak Syans Agrikiltirèl / Inivèsité Laflorid / Larry R. Arrington, Dirèktè.

Pwogram Kado nan Depatéman Laflorid sou Afè Kontwol Konstriksyon Rezidans nan Kominoté-a péyé yon pati nan piblikasyon-an. 


\section{Mete Sipò Oubyen Chanjé Pòt Remize-La}

Si ou rete nan yon kay ki ansyen ou fèt pou mete sipò ouswa chanjé pòt remize-la. Nou gen dwa mete sipò antravè enstalé nan mitan chak panno pòt-la pou ranfòse pòt ki la deja-a.

Pou plis fòs, ou gen dwa tou enstalé sipò vètikal ki kapab kouvri tout longè pòt-la. Sipòyo gen pou byen rantré atè-a é anlè pòt-la nan miray-la. Sipò sa-yo gen dwa sòti lè ou pa bezwen li.

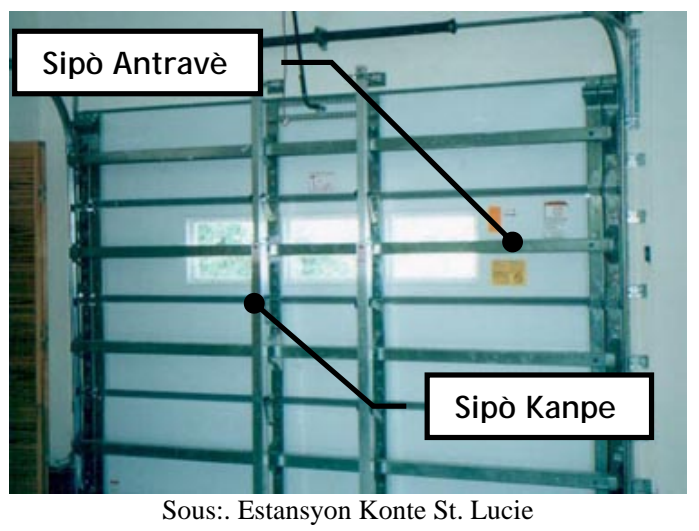

Trak pou pòt remize sa-yo kapab yon lòt gwo pwoblem kap bezwen sipò tou. Jan ke li parèt nan foto ki anba paj-la sou bò dwat-la, yon trak ki pa gen sipò gen dwa ployé. Lè sa-a pòt remize-la gen dwa soti nan miray-la.

\section{Lòt Bagay Pou Nou Konsidéré}

Pafwa, ésaye ranje ansyen pòt rémize pou fè yo pi rezistan pou van gen dwa yon jòb ki trè difisil. Li ta fè plis sans si ou ta bay kontraktè kalifyé enstalé sipò nesésè ouswa chanjé pòt remize-la.

Si ou ta déside fè travy-la oumemm, pa blyé ke pòt-la pral vini pi lou. Sa kapab yon rézon pou chanjé motè ki pou ouvri pòt-la.

Lè na pé mété sipò nan pòt remize-la, sonjé pou ou kouvri tout fenèt ki sous pòt-la ak panno tòl pou pwotéjé vit-la. Piga achté pòt remize ki genyen vit ladan yo.
Si sé pwoteksyon ki piwo-a ké ou vlé, fòk ou achté pwodwi ké Konté Miami-Dade di ke ou kapab itilizé (gadé nan adrès pwodwi ke nou bay anba-la).

\section{Lòt Resous}

Alyans Federal Pou Kay Ak Sekirité www.flash.org

Federal Emergency Management Agency | Prevansyon Pou Dezast \& Bibliotèk Pou Preparasyon www.fema.gov/library/prepandprev.shtm

Enstiti pou Sekirité Biznis ak Kay www.ibhs.org

Konté Miami-Dade | Obeyisans Règ Bilding Pou Chèché Pwodwi

http://www.miamidade.gov/buildingcode/onlin e_product_search.asp

\section{PWOBLÈM}

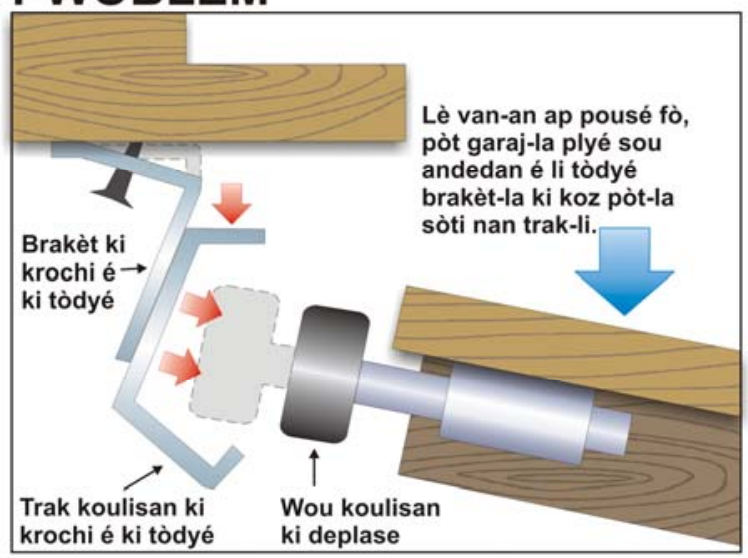

\section{SOLISYON}

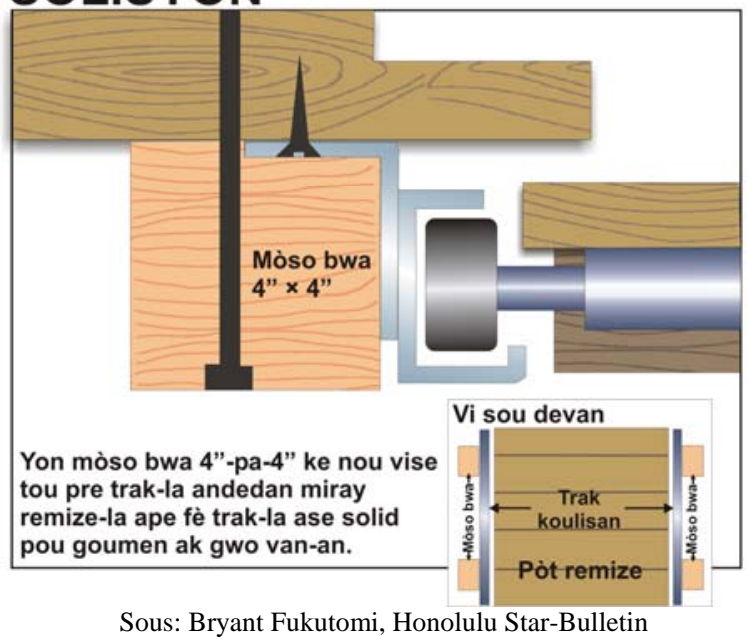

